\title{
HIGHLIGHTS
}

\section{Success stories}

Adoptive T cell therapy has limitations on the extent of cell expansion and persistence, and in the number of cancer types in which it is effective. Mechanistic analysis of exceptional responders in clinical trials may be useful to improve it. Two cases have been reported, showing that deletion of the methylcytosine dioxygenase TET2 enhanced antitumour T cell activity in chronic lymphocytic leukaemia (CLL) and neoantigen-specific tumour-infiltrating lymphocytes (TILs) were efficiently identified and expanded to treat advanced epithelial cancer with a typically low mutation rate, respectively.

Fraietta et al., who published their findings in Nature, identified a patient with CLL who has shown complete and sustained remission following two infusions of autologous chimeric antigen receptor (CAR) T cells targeting the B-lymphocyte antigen CD19 (CTL019 cells). After the first infusion, the patient experienced high fever and signs of cytokine release syndrome (CRS) but no signs of remission. However, after the second infusion was performed to bypass potential CTL019 inhibitory effects by blocking interleukin- 6 (IL-6) to treat the CRS, the onset of CRS and high fever coincided with CLL clearance.

Deep sequencing of the CTL019 cell population revealed that $94 \%$ of the repertoire of the CD8+CTL019 $\mathrm{T}$ cell receptor (TCR) $\beta$-variable region (TRBV) after the second infusion consisted of one single clone, which was not detected before therapy initiation or after the first infusion. The corresponding CTL019 cell clone population had a CAR lentiviral integration site in the TET2 gene, resulting in transcription of chimeric mRNAs and translation of a truncated, functionally ablated TET2 fusion protein. Surprisingly, both CAR+ and CAR- T cell populations expressed a missense variant of TET2, leading to reduced enzymatic function of the TET2 mutant protein compared with the wild-type protein - thus CAR+ T cells in this patient were affected not only by heterozygous loss of function of TET2 on the allele that received the CAR insertion, but also by a hypomorphic TET2 variant on the other, intact allele. The biallelic TET2 dysfunction in CAR $+\mathrm{T}$ cells led to changes in the epigenome characterized by higher chromatin accessibility of genes regulating $\mathrm{T}$ cell effector differentiation and exhaustion, and a less differentiated state compared with the monoallelic TET2 dysfunction in CAR- T cells. In line with this, healthy donor CTL019 cells with TET2 knockdown continuously expanded and sustained the production of cytokines in response to antigen and co-stimulatory signals.

Zacharakis et al., who published their findings in Nature Medicine, described a patient with chemorefractory, hormone receptor-positive metastatic breast cancer. Following adoptive transfer of neoantigen-reactive autologous TILs, all target lesions disappeared 1 year after TIL transfer, and the patient has shown complete and sustained remission. TILs were derived from cultured tumour fragments of the patient's breast cancer and screened against a range of mutations found in the target tumour. TILs were identified with reactivity against mutant versions of solute carrier family 3 member 2 (SLC3A2) and proteasome adapter and scaffold protein ECM29. TRBV-based sorting and single-cell sequencing of TILs stimulated with mutant

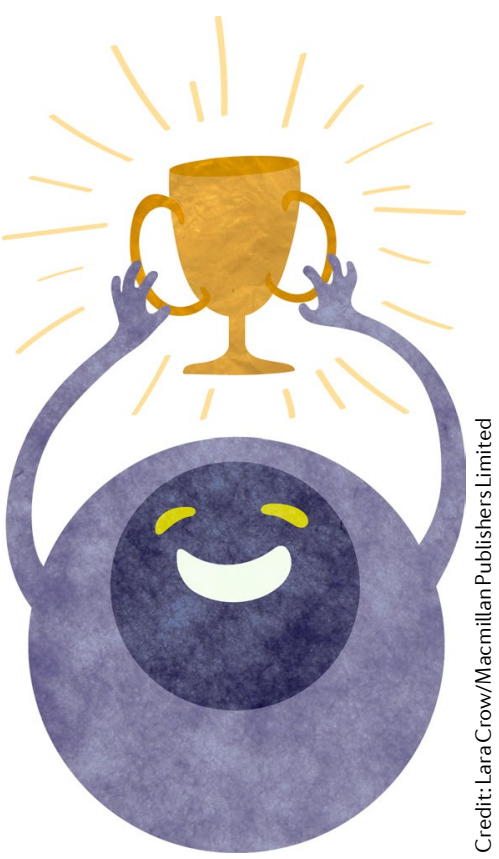

protein-loaded antigen-presenting cells identified seven TCR clonotypes recognising SLC3A2 mutants, and one TCR clonotype recognising mutant ECM29.

TILs derived from the reactive fragments were expanded and infused into the patient, leading to $52 \%$ reduction of the target tumour burden 6 weeks after treatment. Subsequently, analysis of peripheral blood revealed persistence of the neoantigen-reactive TCR clonotypes, and presence of additional TCR clonotypes reactive against mutant calcium-dependent secretion activator 2 and cathepsin B.

Once validated, these studies could open up the potential for targeting TET2 and the epigenome of CAR T cells, and for more efficient identification and expansion of neoantigen-reactive TILs, which can be used to improve effectiveness of adoptive $\mathrm{T}$ cell immunotherapy.

Ulrike Harjes

ORIGINAL ARTICLES Fraietta, J. A. et a Disruption of TET2 promotes the therapeutic efficacy of CD19-targeted T cells. Nature 558 , 307-312 (2018) | Zacharakis, N. et al. Immune recognition of somatic mutations leading to complete durable regression in metastatic breast cancer. Nat. Med. 24, 724-730 (2018) 